\title{
Diversity of the p70 Killer Cell Inhibitory Receptor (KIR3DL) Family Members in a Single Individual
}

\author{
Daeho Kwon, Yong-Joon Chwae, In Hong Choi, Jeon Han Park, Se Jong Kim, and Jongsun Kim* \\ Department of Microbiology and Institute for Immunology and Immunological Diseases, Yonsei University College of Medicine, Seoul \\ 120-752, Korea.
}

(Received on September 21, 1999)

NK cells and some $T$ cells express members of a multigenic family of killer cell inhibitory receptors (KIRs) including p70 KIR (KIR3DL) and p58 KIR (KIR2DL) family that recognize polymorphic class I MHC molecules on target cells and transmit an inhibitory signal to prevent killer cell-mediated cytoxicity. The cDNA sequences of p70 KIR family members reported so far suggest that the p70 KIR gene consists of a multigene complex and that each gene may exhibit certain degrees of polymorphism. However, it is not clear how diverse the repertoire of the p70 KIR family is, particularly in a single individual. To address this question in more detail and to determine some indication as to the origin of the diversity, we cloned p70 KIR cDNAs from a single individual. We identified nine new KIRs that are different from the previously reported ones. A comparison of the amino acid sequences with published sequences of p70 KIRs showed that two clones belonged to the KIR3DL1 family, five clones belonged to the KIR3DL2 family, one clone belonged to the KIR2DL4 family, and one clone appeared to be an alternatively spliced form of p70 KIR. These results suggested that the repertoire of $p 70$ KIR family members in a single individual is highly diverse. It is not clear how the diverse receptors are generated in a single individual, but a comparison of amino acid sequences of p70 KIR family members suggested that some of them may be encoded by distinct genes or their alleles, while others may be generated by a recombination mechanism and/or an alternative splicing mechanism at the maturation of the mRNA transcripts.

Keywords: Alternative Splicing; NK Cells; p70 KIR; Polymorphism; Receptor Repertoire.

\footnotetext{
* To whom correspondence should be addressed.

Tel: 82-2-361-5277; Fax: 82-2-392-7088

E-mail: jkim63@yumc.yonsei.ac.kr
}

\section{Introduction}

NK cells and some $\mathrm{T}$ cells express members of a multigenic family of killer cell inhibitory receptors (KIRs) that recognize polymorphic class I MHC molecules on target cells (Lanier and Phillips, 1996; Lanier et al., 1997; reviewed in Moretta et al., 1997). Both natural cytotoxicity and antibody-dependent cell cytotoxicity of NK cells, as well as CD3/TCR-dependent cytotoxicity of $\mathrm{T}$ cells are inhibited by the co-engagement of KIRs and activating receptors such as FcR and TCR (Cambiaggi et al., 1996; Mingari et al., 1995; Phillips et al., 1995; Vitale et al., 1995). KIRs are type I transmembrane glycoproteins belonging to the Ig superfamily and consist of either two (for p58 KIR and KIR103: KIR2D family) or three (for p70 KIR: KIR3D family) extracellular Ig-related domains, a transmembrane part and a cytoplasmic tail (Colonna and Samaridis, 1995; D'Andrea et al., 1995; Selvakumar et al., 1996; Wagtmann et al., 1995a). The extracellular domain of KIR specifically recognizes particular class I MHC molecules expressed on target cells (Fan et al., 1996; Kim et al., 1997; Rojo et al., 1997; Wagtmann et al., 1995b). The cytoplasmic tail of KIR contains immunoreceptor tyrosine-based inhibition motifs (ITIMs) that function in triggering an inhibitory signal transduction that, in turn, prevents killer cell-mediated cytotoxicity (Binstadt et al., 1996; Burshtyn et al., 1996; Campbell et al., 1996; Fry et al., 1996).

Based on the structures of the extracellular domain and cytoplasmic tail, KIRs can be divided into 5 families, p70, p70 $\mathrm{CYT}$, p58, KIR103, and p50, which can be further classified into 12 subfamilies according to the amino acid sequence homology (Selvakumar et al., 1997; Steffens et al., 1998). The p70 family comprises 2 subfamilies, KIR3DL1-2, which are inhibitory receptors with three

Abbreviations: Ig, immunoglobulin; ITIM, immune receptor tyrosine based inhibition motif; KIR, killer cell inhibitory receptor. 
extracellular Ig domains and two ITIMs in the cytoplasmic tail; the p70 $\triangle$ CYT family comprises 1 subfamily, KIR3DS1, which is a noninhibitory receptor with three extracellular Ig domains and a short cytoplasmic tail lacking any ITIM; the p58 family comprises 3 subfamilies, KIR2DL1-3, which are inhibitory receptors with two extracellular Ig domains and two ITIMs in the cytoplasmic tail; the KIR103 family comprises 1 subfamily, KIR2DL4, which is an inhibitory receptor with two extracellular Ig domains and a single ITIM in the cytoplasmic tail; the p50 family comprises 5 subfamilies, KIR2DS1-5, which are noninhibitory receptors with two extracellular Ig domains and a short cytoplasmic tail lacking any ITIM. The 12 subfamilies of KIR are thought to be encoded by distinct genes (Parham, 1997; Steffens et al., 1998), and the results from genomic Southern blot and chromosome in situ hybridization analyses also indicate the existence of multiple KIR genes on human chromosome 19q13.4 (Suto et al., 1996; 1998).

The cDNA sequences of KIR family members reported so far suggest that the KIR gene consists of a multigene complex and that each gene may exhibit certain degrees of polymorphism (Parham, 1997; Steffens et al., 1998). For example, nine distinct KIR3DL1-like transcripts and five distinct KIR3DL2-like transcripts have been reported so far (Steffens et al., 1998; Vyas et al., 1998), although the relationship between these cDNAs remains unknown. Furthermore, it is not clear how diverse the repertoire of the p70 KIR family is, particularly in a single individual. To address this question in more detail and to determine some indication as to the origin of the diversity, we cloned p70 KIR cDNAs from a single individual by taking advantage of PCR amplification. DNA sequencing analysis of the clones revealed that the repertoire of p70 KIR family members in a single individual is highly diverse. It is not clear how the diverse receptors are produced in a single individual, but a comparison of amino acid sequences of p70 KIR family members suggested that some of them may be encoded by distinct genes or their alleles, while others may be generated by a recombination mechanism at the maturation of the mRNA transcripts.

\section{Materials and Methods}

Total RNA isolation and cDNA synthesis PBMCs were purified by Ficoll-Hypaque (Lymphoprep, Nyegaard, Olso, Norway) density gradient centrifugation from $10 \mathrm{ml}$ of heparinzed peripheral blood donated by a healthy volunteer. Total RNA was isolated by using RNeasy kit (Qiagen, Santa Claris, CA). The first strand cDNA of the total RNA was synthesized by using $5 \mu \mathrm{g}$ of RNA, 0.1 OD U of random hexamer (Pharmacia, Uppsala, Sweden) and $500 \mathrm{U}$ of murine leukemia virus reverse transcriptase (Life Technologies, Gaithersbrug, MD) in a total volume of $100 \mu \mathrm{l}$. The reaction mixture was incubated for $1 \mathrm{~h}$ at $42^{\circ} \mathrm{C}$ and used for PCR without further manipulation.
cDNA cloning of the extracellular domain of p70 KIRs The protein-coding region of the extracellular domain of p70 KIR (amino acid 1-297) was amplified by PCR using $10 \mu \mathrm{l}$ of cDNA and Taq DNA polymerase with the 5 '-oligonucleotide primer TTGTTGAATTCTAAGGAGGATATTAAAATGCACGTGGGTGGTCAGGACAA and 3'-oligonucleotide primer GTTTCAAAGCTTTAGTTTCCTGTGACAGAAACAAG. The oligonucleotide primers were designed to match polymorphic positions common to KIR3DLs, as determined from an alignment of different KIR3DL sequences (Steffens et al., 1998). The 5' primer encoded an N-terminal methionine and the first 7 amino acids of the protein. The stop codon TAA at codon 298 was included in the HindIII recognition sites of the $3^{\prime}$ primer. The amplified cDNAs were gel purified, digested with EcoRI/HindIII, and ligated into an E. coli expression vector pLM1 (Kim et al., 1996; Sodeoka et al., 1993) that had been digested with EcoRI/ HindIII and gel purified. The recombinant DNAs were transformed into the $E$. coli strain $\mathrm{XL}-1$ blue, and clones containing the correct insert were identified by restriction digest followed by agarose gel electrophoresis. The inserts were sequenced in both orientations by using the dideoxy chain termination technique with the Sequenase 2.0 Kit (United States Biochemicals, Cleveland, $\mathrm{OH}$ ) and by using an automated nucleic acid sequencer (ABI Prism, USA).

Southern blot analysis Southern blot analysis of the PCR product was performed using the $\mathrm{ECL}^{\mathrm{TM}}$ system (Amersham, Little Chalfont, UK) following the manufacturer's recommendations. Briefly, the amplified DNAs were analyzed by electrophoresis in a $1.0 \%$ agarose gel and transferred to a Hybond $^{\mathrm{TM}}-\mathrm{N}^{+}$membrane (Amersham, Little Chalfont, UK). Prehybridization was performed for $1 \mathrm{~h}$ in prehybridization buffer. After hybridization with horse radish peroxidase-labeled p70 KIR cDNA (NKB1) probe for $24 \mathrm{~h}$ at $42^{\circ} \mathrm{C}$, the membrane was washed and exposed to X-ray film at room temperature.

Amino acid sequence analysis Amino acid sequences of p70 KIR family members were obtained from the GenBank and the sequences of the extracellular Ig domains were compared with our new p70 KIR clones. When multiple identical sequences were available in the database, only one representative sequence was selected and used for clarity in the sequence comparison.

\section{Results}

cDNA cloning of p70 KIRs from PBMCs It is important to determine how diverse the repertoire of the KIR family is, particularly in a single individual. To address this issue, we cloned cDNA of the p70 KIR family from a single individual. The protein coding region of the extracellular domain of p70 KIR ( $900 \mathrm{bp})$ was amplified by PCR using cDNA made from PBMCs as a template. Interestingly, we found that the PCR product appeared as one major band ( $900 \mathrm{bp})$ and one weak band ( $600 \mathrm{bp})$ on an agarose gel. The two bands were hybridized with the p70 KIR cDNA probe, indicating that the bands were derived from p70 KIR genes (data not shown). The amplified DNAs were subcloned into an $E$. coli expression 
vector, and clones containing the correct insert were identified by agarose gel electrophoresis. DNA sequencing analysis of the clones revealed that the $900 \mathrm{bp} \mathrm{PCR}$ product results from the amplification of $\mathrm{p} 70$ KIRs (Figs. 1 and 3), while the $600 \mathrm{bp}$ PCR results from the amplification of KIR103 (data not shown)

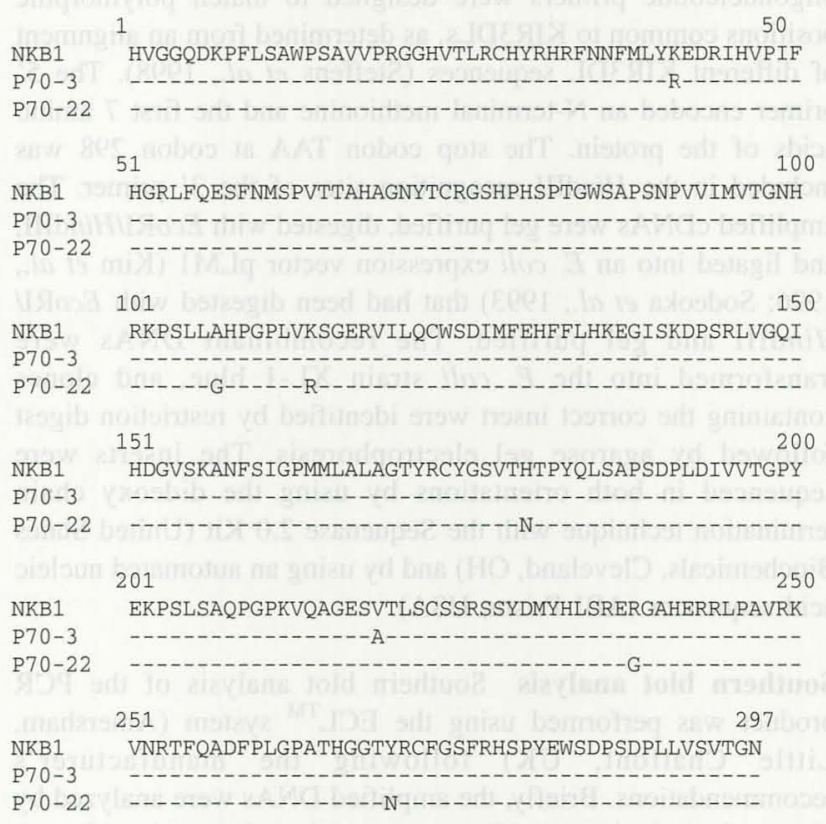

Fig. 1. Amino acid sequence alignment of the KIR3DL1 family members identified in this study. Deduced amino acid sequences of KIR3DL1 family members are aligned manually with published sequences of NKB1 (D'Andrea et al., 1995). Amino acid residues are numbered after the point of signal sequence cleavage and indicated by single-letter codes. Amino acid identity is indicated by dashes. Sequences for the first 7 amino acids and the last 7 amino acids may be primed by primer sequences. cDNA sequences of p70-3 and p70-22 have been deposited in GenBank with the accession numbers AF208688 and AF208689, respectively.

\begin{tabular}{|c|c|c|c|c|c|c|c|c|c|c|c|c|c|c|}
\hline a.a residue & 29 & 41 & 47 & 54 & 107 & 114 & 120 & 140 & 145 & 150 & 180 & 219 & 238 & 270 \\
\hline KIR3DL1 & V F & $\mathrm{K}$ & $\mathrm{V}$ & L & A & K & I & S & $\mathrm{R}$ & I & $\mathrm{H}$ & V & $\mathrm{R}$ & $\mathrm{Y}$ \\
\hline JME1 & - & - & - & - & - & - & - & - & S & - & - & - & G & - \\
\hline JME2 & $-s$ & - & - & - & - & - & $\mathrm{T}$ & - & S & - & - & - & G & - \\
\hline JME3 & - & - & - & - & - & - & - & - & - & - & - & - & - & - \\
\hline JME 4 & - & - & - & - & - & - & - & P & S & $\mathrm{T}$ & - & - & G & - \\
\hline JME5 & - & - & - & - & - & - & - & - & S & - & - & - & G & - \\
\hline JME6 & - & - & - & - & - & - & - & - & - & - & - & - & G & - \\
\hline KIR3DL1V & - & - & - & - & - & - & - & - & - & - & - & - & G & - \\
\hline NKAT3 & M - & - & I & I & - & - & - & - & - & - & - & - & G & - \\
\hline P70-3 & - & R & - & - & - & - & - & - & - & - & - & A & - & - \\
\hline P70-22 & - & - & - & - & G & $\mathrm{R}$ & - & - & - & - & N & & G & $\mathrm{N}$ \\
\hline
\end{tabular}

Fig. 2. Amino acid sequence differences between KIR3DL1 family members and KIR3DL1 (NKB1) in the extracellular Ig domains. Only amino acids with changes are shown, and all other sequences are identical to KIR3DL1. The sequence diversity of each KIR3DL1 family member is scattered over the three Ig domains. Amino acid sequences of KIR3DL1, KIR3DL1v and NKAT3 were referred from Steffens et al. (1998), and those of JME1-6 were referred from Vyas et al. (1998).

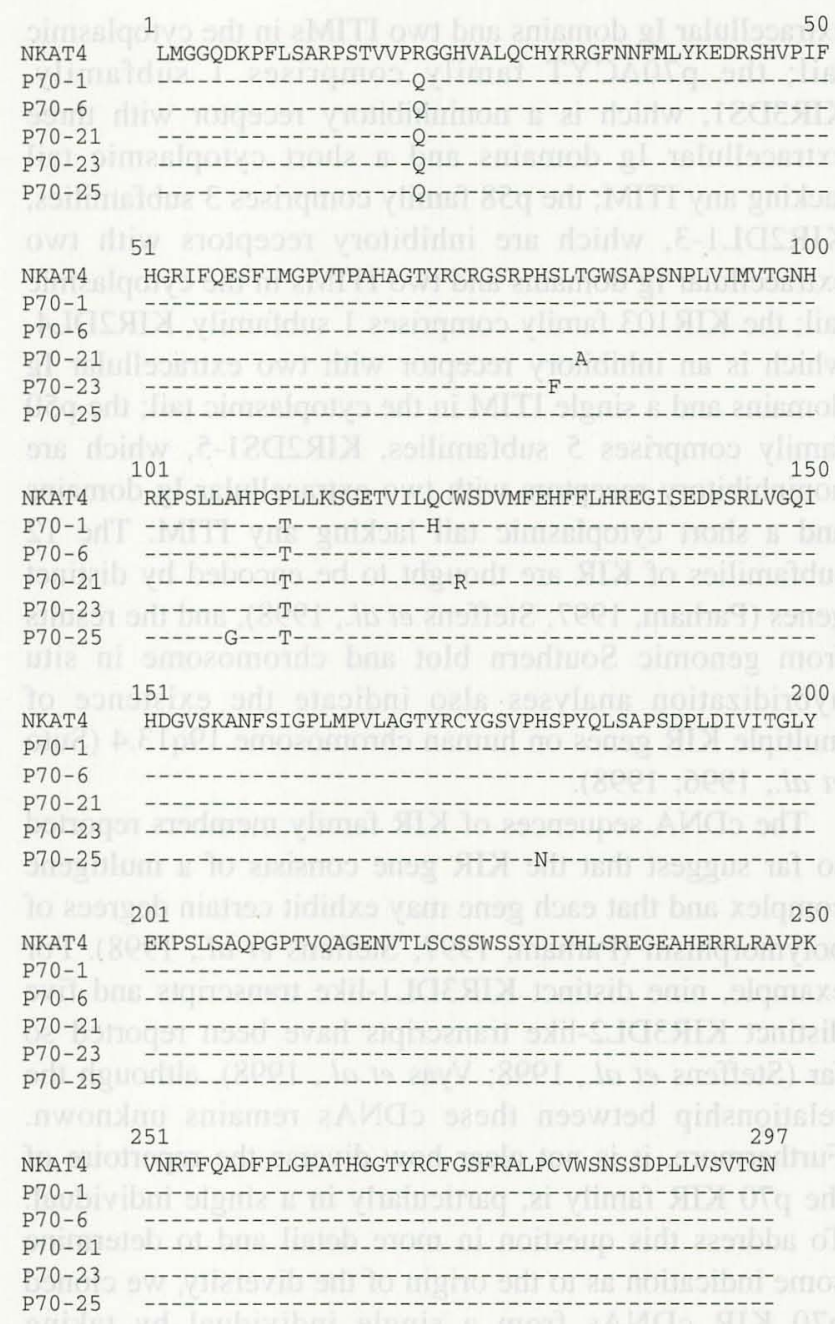

Fig. 3. Amino acid sequence alignment of the KIR3DL2 family members identified in this study. As in Fig. 2, deduced amino acid sequences of KIR3DL2 family members are aligned manually with published sequences of NKAT4 (Colonna \& Samaridis, 1995). cDNA sequences of p70-1, p70-6, p70-21, p70-23, and p70-25 have been deposited in GenBank with the accession numbers AF208683, AF208684, AF208685, AF208686, and AF208687, respectively.

Twenty clones were sequenced and nine clones appeared to be independent (p70-1, -3, -6, -21, -22, -23, $-24,-25$, and -32 ), with sequence identities of $85-99 \%$. The amino acid sequences of the 9 newly identified p70 KIRs were different from the previously reported ones in the literature or in the sequence data bases. A comparison of amino acid sequences with published sequences of $\mathrm{p} 70$ KIR showed that two clones were homologous to NKB1 (KIR3DL1 family; Fig. 1), 5 clones were homologous to NKAT4 (KIR3DL2 family; Fig. 3), one clone was homologous to KIR103 (KIR2DL4 family; data not shown), and one clone appeared to be an alternatively spliced form of p70 KIR (Fig. 5). It turned out that the sequence diversity of each p70 KIR clone was scattered over the extracellular Ig domains. 
Diversity of KIR3DL1 family members in a single individual In this study, we identified two different cDNA clones (p70-3, and -22) of the KIR3DL1 family from a Korean blood donor (Fig. 1). The amino acid sequences of both p70-3 and p70-22 are very homologous to that of NKB1 (D'andrea et al., 1995), a representative member of the KIR3DL1 family, with sequence identity of about $98-99 \%$ (Fig. 1), but they are distinct from that of NKAT4 (Colonna and Sammaridis, 1995), a representative member of the KIR3DL2 family, with sequence identity of about $85 \%$. Two amino acids (residue number 41 and 219 ) are substituted in the extracellular Ig domains of p70-3, and five amino acids (residue number 107, 114, 180, 238 and 270) are substituted in the extracellular Ig domains of p70-3. Nine cDNA sequences of NKB1 family members have been reported in the literature or in the sequence data bases (Steffens et al., 1998; Vyas et al., 1998), but the amino acid sequences of p70-3 and -22 are different from the previously reported ones (Fig. 2). This suggests that the repertoire of the KIR3DL1 family is highly diverse among different individuals. However, these eleven KIR3DL1 family members are very similar and their variability is limited to 17 positions in the extracellular Ig domains (Fig. 2). The relationship between these cDNA sequences of KIR3DL1 family members is unclear, but they seem to represent allelic polymorphism of KIR3DL1. If this is true, p70-3 and p70-22 may represent two different allelic forms of NKB1 in a single individual.

Diversity of KIR3DL2 family members in a single individual We also identified 5 cDNAs of the KIR3DL2 family (p70-1, -6, -21, -23, and -25) from a single individual (Fig. 3) whose amino acid sequences were distinct from those of KIR3DL2 family members reported so far. As shown in Fig. 3, the amino acid sequences of these clones are homologous to each other, with substitution of two to four amino acids, and these sequences are very similar to that of NKAT4 (Colonna and Samaridis, 1995), a representative member of the KIR3DL2 family. Compared to the amino acid sequence of NKAT4, three amino acids are substituted in the extracellular Ig domains of p70-1; two amino acids are substituted in the p70-6; four amino acids are substituted in the $\mathrm{p} 70-21$; three amino acids are substituted in the p70-23; and four amino acids are substituted in the p70-25. Interestingly, all the amino acid substitutions occurred in the first two Ig domains. The origin of these homologous clones is not clear, but the presence of multiple clones of the KIR3DL2 family suggests that the repertoire of KIR3DL2 mRNA transcripts is also diverse in a single individual.

Five different full-length genes of the KIR3DL2 family have been reported so far (Steffens et al., 1998). The members of KIR3DL2 vary with respect to their degree of polymorphism compared to NKAT4. NKAT4A, NKAT4B, and AMC5 differ by 1 or 2 amino acids, while cl-17.1c

$\begin{array}{lccccccccccccc}\text { a.a residue } & 19 & 20 & 81 & 83 & 92 & 107 & 111 & 122 & 124 & 137 & 145 & 180 & 207 \\ \text { KIR3DL2 } & \text { P } & \text { R } & \text { S } & \text { T } & \text { I } & \text { A } & \text { P } & \text { Q } & \text { W } & \text { E } & \text { R } & \text { H } & \text { A } \\ \text { NKAT4A } & - & - & - & - & \text { V } & - & - & - & - & - & - & - & - \\ \text { NKAT4B } & - & - & - & - & \text { V } & - & - & - & - & - & \text { H } & - & - \\ \text { AMC5 } & - & - & - & - & \text { L } & - & - & - & - & \text { D } & - & - & - \\ \text { C1-17.1C } & \text { A } & - & - & - & \text { V } & - & - & - & - & - & \text { H } & - & \text { P } \\ \text { P70-1 } & - & \text { Q } & - & - & - & - & \text { T } & \text { H } & - & - & - & - & - \\ \text { P70-6 } & - & \text { Q } & - & - & - & - & \text { T } & - & - & - & - & - & - \\ \text { P70-21 } & - & \text { Q } & - & \text { A } & - & - & \text { T } & - & \text { R } & - & - & - & - \\ \text { P70-23 } & - & \text { Q } & \text { F } & - & - & - & \text { T } & - & - & - & - & - & - \\ \text { P70-25 } & - & \text { Q } & - & - & - & \text { G } & \text { T } & - & - & - & - & \text { N } & -\end{array}$

Fig. 4. Amino acid sequence differences between KIR3DL2 family members and KIR3DL2 (NKAT4) in the extracellular Ig domains. Only amino acids with changes are shown, and all other sequences are identical to KIR3DL2. Unlike the case of KIR3DL1 family members, most of the amino acid substitutions occurred in the first and second Ig domains. Amino acid sequences of KIR3DL2, NKAT4A, B, AMC5, and cl-17.1c were referred from Steffens et al. (1998).

shows 5 amino acid substitutions. The newly identified KIR3DL2 family members in this study are different from the previously reported ones (Fig. 4). These results suggest that KIR3DL2 also displays a high degree of polymorphism among different individuals. It will be interesting to investigate the functional significance of the polymorphism of the KIR3DL2 family.

Alternative splicing forms of p70 KIR Alternative splicing of the p70 KIR gene was previously suggested by the cloning of a cDNA variant of NKB1 (NKB1B) which lacks 17 amino acids in the stem region close to the transmembrane domain (codon 299-315; D'Andrea et al., 1995). In addition to this clone, 2 alternatively spliced products of p70 KIR have been reported (D'hring et al., 1996; Pende et al., 1996). NKAT3-delta $\operatorname{Ig} 1$ is an alternatively spliced product of NKAT3 and shows complete deletion of the first Ig domain. $8.11 \mathrm{c}$ is an alternatively spliced product of an as yet unidentified full length gene of the KIR3DL2 family. It has four distinct amino acid changes compared to NKAT4 and shows a deletion of 17 amino acids in the stem region (codon 297313). In this study, we also detected a truncated form of $p 70$ KIR (p70-24), which has a deletion of 29 amino acids in the junction between the second and third Ig domains (codon 183-211, Fig. 5). As shown in Fig. 5, p70-24 is distinct from other alternatively spliced forms. Our results more clearly document that the repertoire of p70 KIR is also diverse in size, probably due to the alternative splicing mechanism.

\section{Discussion}

We describe here two novel cDNA clones of the KIR3DL1 family and five novel cDNA clones of the KIR3DL2 family of NK receptors. Previously, nine KIR3DL1-like transcripts and five KIR-3DL2-like transcripts were 


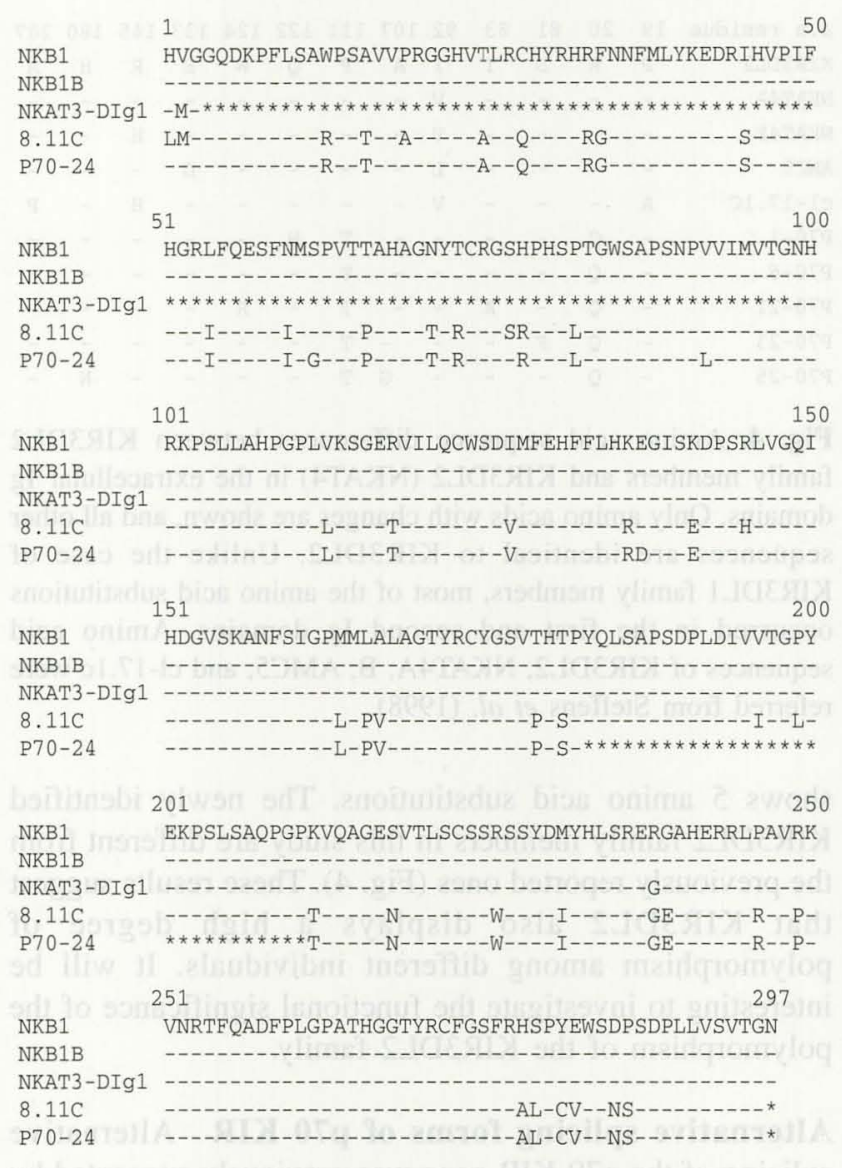

Fig. 5. Amino acid sequence alignment of the alternatively spliced forms of p70 KIR family. Deduced amino acid sequences of p70-24 is aligned manually with published sequences of NKB1 and three previously reported alternatively spliced forms of $\mathrm{p} 70$ KIR. NKB1B (D'Andrea et al., 1995) and 8.11c (Pende et al., 1996) lack 17 amino acids in the stem region close to the transmembrane domain (codon 299-315 for NKB1B and 297-313 for $8.11 \mathrm{c}$ ), and NKAT3-delta Ig 1 has complete deletion of the first Ig domain (Döhring et al., 1996). Amino acid identity is indicated by dashes, and deleted amino acids are indicated by asterisks. Amino acid sequences of NKB1B, NKAT3-delta Ig1 and 8.11c are taken from GenBank (accession numbers U33328, L76664 and X93596, respectively). cDNA sequence of p70-24 has been deposited in GenBank with the accession numbers AF208690.

reported. Together with the previously reported KIR3DL family members, these 11 KIR3DL1-like sequences and 10 KIR3DL2-like sequences constitute very closely related KIR families with a few amino acid substitutions. These results suggest that the repertoires of both the KIR3DL1 family and the KIR3DL2 family are highly diverse with extensive polymorphism within the genes, although the origin of the diversity remains unclear.

Recently, Vyas et al. (1998) identified six new cDNA clones of KIR3DL1 family members and compared the deduced amino acid sequences with the previously reported KIR3DL1 sequences, including NKB1, KIR3DL1v and NKAT3. These nine KIR3DL1 transcripts are very similar and their variability is limited to only 19 codon positions among 423 amino acids. These studies demonstrated that the KIR3DL1 gene family encodes a large number of different transcripts. Based on the amino acid sequence variation patterns of the transcripts, they proposed that four transcripts could be encoded by different KIR3DL1 genes, while the others would be generated by a recombination process. Similarly, five different mRNA transcripts of the KIR3DL2 family have been reported, but the origin of the diversity remains unknown. However, our new KIR3DL2-like transcripts provide evidence for the role of genetic recombination in the generation of KIR3DL2 diversity, too.

Genetic recombination as a mechanism for KIR diversification has been proposed based on KIR sequence analysis (Valiante et al., 1997). A comparison of KIR sequences reveals a "patch-work" pattern of variability in which most substitutions are found in more than one sequence (Valiante et al., 1997). This pattern is analogous to that seen for highly polymorphic MHC genes (Parham et al., 1995). Like the case of MHC genes, the evidence for recombination as a mechanism for KIR diversification has been demonstrated by discoveries of hybrid p58 KIR sequences (Chwae et al., 1999; Shilling et al., 1998). The hybrid clones, which exhibit different amino acid sequence identity with two distinct kinds of KIR at the N-terminal and C-terminal domains, respectively, define potential events of recombination during the evolution of KIR genes and/or at the maturation of KIR mRNA transcripts.

In addition to the 7 distinct KIR3DL family members, a truncated isoform of KIR3DL1 (p70-24) was identified in this study. Previously, three alternative splicing forms of the KIR3DL family (NKB1B, 8.11c and NKAT3-delta Ig1) were reported. NKB1B (D'Andrea et al., 1995) and 8.11c (Pende et al., 1996), which are isoforms of NKB1 and an as yet unidentified full length gene, respectively, lack 17 amino acids in the stem region close to the transmembrane domain (codon 299-315 for NKB1B and 297-313 for 8.11c). NKAT3-delta Ig1 is an alternatively spliced product of NKAT3 and shows complete deletion of the first Ig domain (Döhring et al., 1996). Interestingly, p70-24 has a deletion of 29 amino acids in the junction between the second and third Ig domains (codon 183-211), suggesting that the repertoire of the KIR3DL family is also diverse in size. Alternative splicing forms of the KIR2D family (p58 and p50 KIR) are well documented (Chwae et al., 1999; Döhring et al., 1996). We previously identified four groups of differently spliced forms of p58 KIRs in a single individual (Chwae et al., 1999). The first group of a differently spliced form lacks $1 / 4$ of the first Ig domain and $3 / 4$ of the second Ig domain. A differently spliced isoform of p58 KIR which belongs to this group, as well as other isoforms which lack either the first or second Ig domain, 
have also been reported by Döhring et al. (1996). The second group lacks half of the second Ig domain. The third group lacks $1 / 12$ of the first Ig domain and $1 / 4$ of the second Ig domain. The fourth group lacks $1 / 4$ of the first Ig domain. It is a very fascinating question how the differently spliced isoforms of p58 KIRs could be generated, and the particular DNA sequences found in the p58 KIR cDNAs gave a clue to the answer (Chwae et al., 1999). Remarkably, the splicing sites of the differently spliced isoforms of p58 KIRs were reminiscent of the intron/exon splicing sites (Mount, 1982). Based on this observation, we proposed previously that some part of the exon could be spliced out by an alternative splicing mechanism because of the presence of these particular DNA sequences (potential splice donor and acceptor sequences). Interestingly, those splicing sequences are well conserved, not only in p58 KIRs, but also in p70 KIRs. Therefore, it seems reasonable to assume that the differently spliced forms of the KIR3DL family may also be generated by an alternative splicing of some part of the exon that has the potential splice donor and acceptor sequences.

It is not known whether polymorphism of KIR affects recognition of class I MHC molecules. However, the importance of only a single amino acid substitution has clearly been demonstrated in site-directed mutagenesis experiments of cl-42, a member of the KIR2DL1 family (Winter and Long, 1997). Exchanging a methionine at position 44 of the first Ig domain of cl-42 with a lysine in cl-43, a member of the KIR2DL2 family, at the corresponding position led to switching of the ligand specificity of cl-42 from HLA-Cw4 to HLA-Cw3, and vice versa. A comparison of the amino acid sequences of KIR3DL family members indicates that most of the variants have a few amino acid changes in the extracellular Ig domains (Figs. 1-4). The relationship between these KIR3DL variants is unknown, but they may represent distinct gene products and/or allelic polymorphism. We speculate that a possible function of the diverse repertoire of KIR may be related to the fine-tuning of immune responses, since the KIR-MHC interaction would be affected by a few amino acid changes in KIR variants. It will therefore be important to investigate the functional significance of the KIR3DL variants.

Acknowledgments We thank B. Ross for the critical reading of the manuscript, S. E. Cho for technical assistance, and our colleagues for helpful discussions. This work was supported by a research grant from the Ministry of Education (1997-1998), Korea.

\section{References}

Binstadt, B. A., Brumbaugh, K. M., Dick, C. J., Scharenberg, A. M., Williams, B. L., Colonna, M., Lanier, L. L., Kinet, J. P.,
Abraham, R. T., and Leibson, P. J. (1996) Sequential involvement of Lck and SHP-1 with MHC-recognizing receptors on NK cells inhibits FcR-initiated tyrosine kinase activation. Immunity 5, 629-638.

Burshtyn, D. N., Scharenberg, A. M., Wagtman, N., Rajagopalan, S., Berrada, K., Yi, T., Kinet, J. P., and Long, E. O. (1996) Recruitment of tyrosine phosphatase HCP by the killer cell inhibitory receptor. Immunity 4, 77-85.

Cambiaggi, A., Orengo, A. M., Meazza, R., Sforzini, S., Tazzari, P. L., Laura, F., Raspadori, D., Zambello, R., Semenzato, G., Moretta, L., and Ferrini, S. (1996) The natural killer-related receptor for HLA-C expressed on T cells from $\mathrm{CD}^{+}$ lymphoproliferative disease of granular lymphocytes displays either inhibitory or stimulatory functions. Blood 87, 23692375 .

Campbell, K. S., Dessing, M., Lopez-Botet, M., Cella, M., and Colonna, M. (1996) Tyrosine phosphorylation of a human killer inhibitory receptor recruits protein tyrosine phosphatase 1C. J. Exp. Med. 184, 93-100.

Chwae, Y.-J., Cho, S. E., Kim, S. J., and Kim, J. (1999) Diversity of the repertoire of p58 killer cell inhibitory receptors in a single individual. Immunol. Lett. 68, 267-274.

Colonna, M. and Samaridis, J. (1995) Cloning of immunoglobulin-superfamily members associated with HLA$\mathrm{C}$ and HLA-B recognition by human natural killer cells. Science 268, 405-408.

D'Andrea, A., Chang, C., Franz-Bacon, K., McClanahan, T., Phillips, J. H., and Lanier, L. L. (1995) Molecular cloning of NKB1: a natural killer cell receptor for HLA-B allotypes. $J$. Immunol. 155, 2306-2310.

Döhring, C., Samaridis, J., and Colona, M. (1996) Alternatively spliced forms of human killer inhibitory receptors. Immunogenetics 44, 227-230.

Fan, Q. R., Garboczi, D. N., Winter, C. C., Wagtmann, N., Long, E. O., and Wiley, D. C. (1996) Direct binding of a soluble natural killer cell inhibitory receptor to a soluble human leukocyte antigen-Cw4 class I major histocompatibility complex molecule. Proc. Natl. Acad. Sci. USA 93, 7178-7183.

Fry, A. M., Lanier, L. L., and Weiss, A. (1996) Phosphotyrosines in the killer cell inhibitory receptor motif of NKB1 are required for negative signaling and for association with protein phosphatase 1C. J. Exp. Med. 184, 295-300.

Kim, J., Chwae, Y. J., Kim, M. Y., Choi, I. H., Park, J. H., and Kim, S. J. (1997) Molecular basis of HLA-C recognition by p58 natural killer cell inhibitory receptors. J. Immunol. 159. 3875-3882.

Kim, J., van Tienhoven, E., Garboczi, D. N., Utz, U., Kim, M. Y., and Wiley, D. C. (1996) Specific binding of a soluble recombinant $\beta$-chain T-cell antigen receptor to Toxic Shock Syndrome Toxin-1, a superantigen. Mol. Cells 6, 590-596.

Lanier, L. L. (1997) NK cells: from no receptor to too many. Immunity 6, 371-378.

Lanier, L. L. and Phillips, J. H. (1996) Inhibitory MHC class I receptors on NK cells and T cells. Immunol. Today 17, 86-91.

Mingari, M. C., Vitale, C., Cambiaggi, A., Schiavetti, F., Melioli, G., Ferrini, S., and Poggi, A. (1995) Cytolytic T lymphocytes displaying natural killer (NK)-like activity: expression of NK-related functional receptors for HLA class I molecules (p58 and CD94) and inhibitory effect on the TCR-mediated target cell lysis or lymphokine production. Int. Immunol. 7, 697-703.

Moretta, A., Biassoni, R., Bottino, C., Pende, D., Vitale, M., Poggi, A., Mingari, M. C., and Moretta, L. (1997) Major histocompatibility complex class-I specific receptors on human natural killer and T lymphocytes. Immunol. Rev. 155, $105-117$. 
Mount, S. M. (1982) A catalogue of splice junction sequences. Nucleic Acids Res. 10, 459-472.

Parham, P. (1997) NK cells, MHC class I antigens and missing self. Immunol. Rev. 155, 5-221.

Parham, P., Adams, E. J., and Arnett, K. L. (1995) The origins of HLA-A, B, C polymorphism. Immunol. Rev. 143, 141-180.

Pende, D., Biassoni, R., Cantoni, C., Verdiani, S., Falco, M., Di Donato, C., Accame, L., Bottino, C., Moretta, A., and Moretta, L. (1996) The natural killer cell receptor specific for HLA-A allotypes: a novel member of the p58/p70 family of inhibitory receptors that is characterized by three immunoglobulin-like domains and is expressed as a $140-\mathrm{kD}$ disulfide-linked dimer. J. Exp. Med. 184, 505-518.

Phillips, J. H., Gumperz, J. E., Parham, P., and Lanier, L. L. (1995) Superantigen-dependent, cell mediated cytotoxicity inhibited by MHC class I receptors on T lymphocytes. Science 268, 403-405.

Rojo, S., Wagtmann, N., and Long, E. O. (1997) Binding of a soluble p70 killer cell inhibitory receptor to HLA-B*5101: requirement for all three p70 immunoglobulin domains. Eur. J. Immunol. 27, 568-571.

Selvakumar, A., Steffens, U., and Dupont, B. (1997) Polymorphism and domain variability of human killer cell inhibitory receptors. Immunol. Rev. 155, 183-196.

Shilling, H. G., Lienert-Weidenbach, K., Valiante, N. M., Uhrberg, M., and Parham, P. (1998) Evidence for recombination as a mechanism for KIR diversification. Immunogenetics 48, 413-416.

Sodeoka, M., Larson, C. J., Chen, L., LeClair, K. P., and Verdine, G. L. (1993) A multifunctional plasmid for protein expression by ECPCR: overproduction of the p50 subunit of NF-KB. Bioorg. Med. Chem. Lett. 3,1089-1093.

Steffens, U., Vyas, Y., Dupont, B., Selvakumar, A. (1998) Nucleotide and amino acid sequence alignment for human killer cell inhibitory receptor (KIR). Tissue Antigens 51, 398413 .
Suto, Y., Maenaka, K., Yabe, T., Hirai, M., Tokunaga, K., Tadok, K., and Juji, T. (1996) Chromosomal localization of the human killer cell class I receptor family genes on $19 \mathrm{q} 13.4$ by fluorescence hybridization. Genomics 35, 270-272.

Suto, Y., Ishikawa, Y., Kasahara, M., Kasai, F., Yabe, T., Akaza, T., and Juji, T. (1998) Gene arrangement of the killer inhibitory receptor family on human chromosome $19 \mathrm{q} 13.4$ detected by fiber-FISH. Immunogenetics 48, 235-241.

Valiante, N. M., Lienert, K., Shilling, H. G., Smits, B. J., and Parham, P. (1997) Killer cell receptors: keeping pace with MHC class I evolution. Immunol. Rev. 155, 155-164.

Vitale, M., Sivori, S., Pende, D., Moretta, L., and Moretta, A. (1995) Coexpression of two functionally independent p58 inhibitory receptors in human natural killer cell clones results in the inability to kill all normal allogenetic target cells. Proc. Natl. Acad. Sci. USA 92, 3536-3540.

Vyas, Y., Selvakumar, A., Steffens, U., and Dupont, B. (1998) Multiple transcripts of the killer cell immunoglobulin-like receptor family, KIR3DL1 (NKB1), are expressed by natural killer cells of a single individual. Tissue Antigens 52, 510-519.

Wagtmann, N., Biassoni, R., Cantoni, C., Verdani, S., Manati, M. S., Vitale, M., Cottino, C., Moretta, L., Moretta, A., and Long, E. O. (1995a) Molecular clones of the p58 NK cell receptor reveal immunoglobulin-related molecules with diversity in both the extra- and intracellular domains. Immunity 2, 439449.

Wagtmann, N., Rajagopalan, S., Winter, C. C., Peruzzi, M., and Long, E. O. (1995b) Killer cell inhibitory receptors specific for HLA-C and HLA-B identified by direct binding and by functional transfer. Immunity 3, 801-809.

Winter, C. and Long, E. O. (1997) A single amino acid in the p58 killer cell inhibitory receptor controls the ability of natural killer cells to discriminate between the two groups of HLA-C allotypes. J. Immunol. 158, 4026-4028. 